\title{
Programa Materno Infantil y Dinámica de Población -3
}

\author{
Planificación Familiar
}

\author{
Dres.: Luis Daza Parada, M.D., Oscar Henao Echeverry. M.D., \\ Jairo Barragán Espinosa. M.D.
}

\section{Introducción}

Los programas de Planificación Familiar en Colombia nacen en el año 1965, dada la iniciativa de dos instituciones del sector privado como son Profamilia y Ascofame y unas pocas del sector oficial.

A pesar de las diferentes limitantes que ha sufrido en su proceso de desarroIlo, han sido muy favorables los resultados obtenidos y el impacto producido sobre los hechos demográficos del país. A comienzos de la década de los 60 , la tasa de natalidad en Colombia, fluctuaba entre 44.0 y $\mathbf{4 5 . 5}$ por mil habitantes, lo cual colocaba al país en el primero o segundo puesto en Latinoamérica, con duplicación de la población cada 17 a 20 años. Posteriormente ésta tasa ha disminuido en forma cada vez más acelerada hasta llegar en 1980 a 28.9 por mil habitantes.

Por otra parte, la fecundidad demostró un descenso del $33 \%$ entre 1968 y 1978 , colocando al país en un lugar preponderante a nivel mundial en cuanto a disminución de la fecundidad se refiere.

Al hacer el análisis del impacto que los programas organizados de planificación familiar han tenido tanto dentro del sector oficial como del privado, se calcula en un $50 \%$ su contribución al descenso de la fecundidad y el $50 \%$ restante a variables tales, como: nivel de educación, cambios en los patrones de nupcialidad, incorporación de la mujer a la fuerza de trabajo, mortalidad, migraciones y otros aspectos de tipo socio-cultural.

\section{Estado Actual del Programa en el Sector Oficial:}

Los Programas de Planificación Familiar en el Ministerio de Salud se han venido extendiendo en forma progresiva a partir de la creación del Programa Materno Infantil en el año 1969. A nivel de las Instituciones propias del Ministerio, se ha implementado la oferta a nivel de Programa, con los siguientes métodos anticonceptivos:
A. Métodos Quirúrgicos:
- Laparoscopia
- Minilaparotomía

B. Dispositivo Intrauterino:

- T de cobre

- Lippes

C. Hormonales:

- Orales

- Inyectables 
D. Locales:

- Condón

- Tabletas vaginales

En el año 1980 fueron reportados al Subsistema de Información un total de 87.399 nuevas aceptantes de Planificación Familiar, las cuales, según su edad, muestran un predominio en el grupo de 20 a 29 años representado por el $58.7 \%$ de los casos, continuando en orden de importancia el grupo de 30 a 39 años con $23.2 \%$ y por último $14.4 \%$ y $3.6 \%$ para los grupos menores de 20 años y de 40 y más años, respectivamente.

Según el número de hijos vivos actualmente, el $51 \%$ de las aceptantes tenían 1 a 2 hijos, siguiéndole en su orden las que tenían 3 a 4 hijos con un $27.3 \%$. El porcentaje más bajo $(1.94 \%)$ de usuarias, fue el de las que no tenían ningún hijo vivo en la actualidad.

La tendencia observada en el transcurso de los años, muestra un incremento de usuarias para el grupo de 20 a 29 años, a expensas de los grupos de mayor edad, así como también un incremento en las mujeres de baja paridad ( 1 a 2 hijos), con tendencia a disminuir en las usuarias de paridad cero.

Teniendo en cuenta el método anticonceptivo prescrito, se encontró la siguiente distribución:

$\begin{array}{lc}\text { Anovulatorios: } & 39.9 \% \\ \text { DIU } & 45.1 \% \\ \text { Esterilización } & 8.9 \% \\ \text { Otros } & 5.7 \%\end{array}$

Esta distribución por método comparada con la observada en años anteriores, muestra una disminución porcentual para anovulatorios y un aumento para el DIU y la Esterilización. Datos obtenidos de las últimas “Encuestas de Prevalencia de Anticonceptivos" muestran un predominio para el país de la esterilización sobre el DIU y de los anovulatorios sobre la esterilización.

\section{Programas Quirúrgicos de Planifica- ción Familiar:}

El alto número de mujeres en alto riesgo reproductivo, la elevada demanda de las usuarias por estos métodos y la necesidad de ofrecer todos los métodos disponibles en la actualidad, motivaron al Ministerio de Salud, en 1979 a implementar el denominado:

A. Plan Nacional de Endoscopia Pélvica y Técnicas Quirúrgicas de Esterilización. Este plan cuyo proceso de desarrollo terminó en junio de 1981, incluyó las siguientes fases:

1. Dotación con equipos de laparoscopia e instrumental para Minilaparotomía a:

- 16 Hospitales Universitarios

- 104 Hospitales Regionales

- 3 Hospitales locales

2. Capacitación a:

- 131 Médicos

- 113 Enfermeras

- 8 Técnicos de mantenimiento de equipos.

3. Creación de un Centro de Mantenimiento de Equipos de Laparoscopia y seis (6) Subcentros, geográficamente distribuidos en el país, para garantizar una revisión preventiva de las unidades cada 2 meses y correctiva cada que lo amerite.

B. Plan Nacional de Minilaparotomía. La oferta de servicios de Planificación Familiar, en la mayoría de las Instituciones del nivel local, llevó al Ministerio a iniciar en julio del presente año, el Plan Nacional de Minilaparotomía. El Plan que tendrá una duración de 3 años para su montaje, prevee la dota- 
ción de 457 hospitales locales existentes en el país, con 2 equipos de minilaparotomía por Institución y la capacitación de 1 médico y 1 enfermera o auxiliar de enfermería en estas técnicas.

C. Centros de Biología de la Reproducción:

Creación de Centros de Biología de la Reproducción a nivel de Hospitales Universitarios, orientados hacia el área de la docencia, asistencia e investigación en el manejo de:

- Diagnóstico y tratamiento para proconcepción a través de:

- Laparoscopia diagnóstica

- Microcirugía

En laparoscopia diagnóstica, adelantada dentro del Plan de Laparoscopia mencionado.

En microcirugía, a través del Plan de Microcirugía Tubárica, adelantado durante 1979 y 1980 , el cual ha

- Capacitado 8 médicos Ginecobstetras del pais en 2 cursos de 2 semanas de duración cada uno, con contenido teórico y de cirugía experimental durante la primera semana y cirugía en pacientes durante la segunda, bajo la tutoría de cirujanos de la Universidad de Johns Hopkins y la Universidad Católica de Chile.

- Dotado 7 centros de microcirugía con instrumental propio para este procedimiento.

- Servicios de anticoncepción:

- Quirúrgicos

- No quirúrgicos

IV. Evaluación de los Programas Quirúrgicos:

La evaluación de estos programas dentro de la División Materno Infantil, se ha realizado en dos (2) formas:

- Directa, a través de visitas a nivel operativo.

- Indirecta, por medio del análisis de encuestas y formularios específicos de esterilización femenina.

\section{A. Encuesta sobre laparoscopia:}

En abril de 1981, se adelantó una encuesta, orientada a la detección de problemas inherentes a la laparoscopia, en la totalidad de las instituciones donde se realizaban estos procedimientos.

Las complicaciones intra-operatorias más frecuentes, reportadas sobre un total de 3.308 laparoscopias realizadas en 1979 y 1980 fueron:

- Desgarro de trompas 53 casos $(16 \%)$.

- Desgarro de cuello uterino 15 casos $(4.5 \%)$.

El total de casos con complicación intra-operatoria fueron 79 , lo cual representa una tasa de $23.9 \%$.

Las complicaciones post-operatorias más frecuentes reportadas sobre igual número de procedimientos son:

- Infección de pared abdominal 39 casos $(11.8 \%)$

- Hemorragia de trompas 20 casos $(6.0 \%)$.

El total de casos reportados con complicación post-operatoria fue de 68 , lo cual representa una tasa de $20.5 \%$.

En resumen, sobre el total de laparoscopias realizadas (3.308), en este período, se observa una tasa más o menos similar de complicaciones intra-operatorias y post-operatorias $(20 \%)$.

Se reportaron durante el primer año de seguimiento del programa, un total de 34 casos de embarazo, lo cual equivale a una tasa de $10.2 \%$. y un caso de mortalidad posterior al procedimiento 
(3\%oo.) atribuible al tipo de anestesia utilizada (ketalar).

B. Características de las usuarias de metodos quirúrgicos de Planificación Familiar:

En lo referente al análisis de formularios de esterilización femenina, se procesaron un total de 6.697 casos, los cuales han permitido al programa conocer no sólo el nivel donde se prestan los servicios y tipo de profesional que efectúa el procedimiento, sino también las características de las usuarias que determinan de acuerdo a lo normado en el Ministerio, el riesgo reproductivo.

Sobre un total de 3.046 formularios con clasificación de riesgo reproductivo, se encontró que la mayor cantidad de casos esterilizados según edad, estaban dentro del grupo de 30 a 39 años. Para el total de esterilizaciones realizadas, el $85.2 \%$ de los casos estaban clasificados en alto y medio riesgo, reproductivo, lo cual está de acuerdo con la norma del Ministerio, dentro del esquema de clasificación del riesgo. El $14.7 \%$ de los casos restantes, clasificados en bajo riesgo, se apartan de la norma y se ha podido establecer a través de supervisión directa o indirecta, que corresponden a grupos de alta edad, paridad, con problemas socio-económicos, como únicas variables ponderables y por otra parte a pacientes de baja edad y paridad con problemas psiquiátricos de tipo oligofrenia y psicosis.

De acuerdo al número de embarazos el promedio más alto (9.8 embarazos por mujer) se observó en el grupo de 40 y más años, siguiéndole en su orden el grupo de 30 a 39 años (7.1 embarazos) y el de 20 a 29 años con un promedio de 5 embarazos por mujer. El promedio de embarazos para el total de casos se encuentra en 6.1 embarazos por mujer, lo cual está de acuerdo a la norma $\mathrm{Na}$ cional. La relación parto-aborto para el total, es de 10 a 1.
Así mismo, tomando en cuenta la edad e hijos actualmente vivos, el porcentaje más elevado de mujeres esterilizadas, se encontró en el grupo de 30 a 39 años con 4 a 6 hijos $(29.3 \%)$ y en el grupo de 20 a 29 años, con igual número de hijos que el anterior.

Teniendo en cuenta la edad y la zona de procedencia, se observó un ligero predominio de aceptantes de la zona urbana $(58.1 \%)$ sobre la zona rural $(41.9 \%)$ con mayores pesos porcentuales en el grupo de 30 a 39 años de edad. Es explicable el predominio de la zona urbana sobre la rural, dado que inicialmente la oferta de servicios y la accesibilidad geográfica, ha sido mejor a nivel urbano. Así mismo el peso porcentual de mujeres en edad fértil, es mayor en la zona urbana. Estadísticas más recientes, sin embargo, nos demuestran una nivelación de aceptantes para las 2 zonas y para todos los métodos anticonceptivos, lo que refleja la mejor difusión de los programas dentro de las áreas rurales.

De acuerdo al nivel educativo, se observa un predominio en los casos que solamente habian cursado su educación primaria en forma completa o incompleta $(71.5 \%)$, seguido por el grupo de mujeres con educación secundaria y más, con un $15 \%$.

El motivo por el cual se realizó la esterilización en el mayor número de casos, está representado por el grupo de 30 a 39 años, por razones socio-económicas $(23.5 \%)$ y combinación de ésta con razones médicas, en un $20.1 \%$. Para el total de casos estudiados, el motivo soció-económico representó el mayor número de casos, equivalente a un $41.4 \%$ del total $y$ en su orden, la combinación de socio-económicas y médicas con un $34.7 \%$.

Del total de casos reportados, el mayor volumen de esterilizaciones fueron realizadas por medio de laparoscopia $(49.2 \%)$ y dentro de este procedimiento 
el tipo de anestesia utilizada fue la analgésica y la anestesia general en proporción similar $(24 \%)$. Para el total de los casos realizados por cualquiera de los procedimientos, la anestesia general predomina con un $52.6 \%$, cifra ésta que se considera elevada principalmente para las laparoscopias, dado que la recomendación del Ministerio en términos generales, es la de utilizar al máximo la analgesia, con el fin de disminuir costos y complicaciones anestésicas en estos procedimientos.

El predominio de la laparoscopia sobre los demás métodos lleva, como es de esperar, a que la técnica de oclusión más frecuente sea la de anillos de silicona $(93.0 \%)$. Dentro de los demás procedimientos realizados diferentes a laparoscopia, la técnica de oclusión más frecuente fue la ligadura y corte de la trompa en el $37.7 \%$ de los casos y bajos porcentajes para la salpinguectomía y fimbriectomía, técnicas éstas que por su radicalidad limitan la posibilidad de reversión del método.

\section{Conclusiones}

1. En la actualidad se ha implementado la oferta de servicios de Planificación Familiar en la casi totalidad de Instituciones del Ministerio de Salud, dando en esta forma una respuesta al incremento de la demanda de usuarias que año por año se ha venido observando.

2. Este incremento en la demanda, tanto para usuarias nuevas, como antiguas en Planificación Familiar, motivó al Ministerio de Salud a delegar funciones en el personal de enfermería, con el fin de mejorar las coberturas $y$ ra- cionalizar la prestación de servicios por niveles dentro del Sistema, todo esto enmarcado dentro del modelo de Riesgo Reproductivo.

3. El modelo de riesgo, además de determinar el método a prescribir, el nivel de atención y el personal responsable de su seguimiento, permite al nivel central y seccional, realizar una mejor auditoría y ajuste del Programa en su marcha.

4. La utilización del modelo de Riesgo Reproductivo, permite una mejor selección del método a prescribir en cada usuaria, especialmente en lo relacionado con los métodos quirúrgicos, limándose en esta forma las asperezas, que se presentaron en algunos sectores frente a estos procedimientos.

5. Los resultados de la encuesta sobre laparoscopia, son muy favorables para el Programa, dada la baja morbilidad y mortalidad reportada, máxime si se toma en cuenta que este procedimiento se evalúa, durante la etapa en la cual los médicos empiezan a adquirir práctica en ésta técnica, que si bien no es nueva, su desconocimiento era casi absoluto.

6. Así mismo, a pesar de ser la laparoscopia una inovación dentro del Programa, la utilización de este procedimiento, ha sido muy favorable en el país, frente a los demás procedimientos existentes.

7. Las características de las usuarias de programas quirúrgicos en Colombia, están de acuerdo con lo normado en el Ministerio de Salud, con base en el modelo de clasificación de Riesgo Reproductivo. 